\title{
SOLVING THE MYSTERIES IN HOT ISM IN EARLY-TYPE
}

\section{GALAXIES}

K. MATSUSHITA, T. OHASHI

Dept. of Phys., Tokyo Metropolitan Univ.

1-1 Minami-Ohsawa, Hachioji, Tokyo 192-03, Japan

AND

K. MAKISHIMA

Dept. of Phys., University of Tokyo

7-3-1, Hongo, Bunkyoku, Tokyo 113, Japan

We have analyzed ASCA data of about 30 early type galaxies, and studied their X-ray emitting ISM (InterStellar Medium) properties. Our study has been motivated by the apparently very low metallicity of the ISM, which cannot easily be reconciled with theoretical predictions. By carefully examining the abundance ratios and uncertainties in the Fe- $\mathrm{L}$ complex, we have concluded that the ISM abundances in X-ray luminous galaxies are in fact about 1 solar. Therefore, the severe discrepancy between the ISM and stellar abundance has been relaxed. The ISM metallicity of $\mathrm{X}$-ray fainter galaxies are uncertain, but at least SNe Ia contribution to the ISM abundance is smaller than in the X-ray luminous ones.

We have also discovered that X-ray emissions from X-ray luminous galaxies are very extended, and expressed with two beta models of different angular scales. This means that the X-ray luminous ellipticals are central galaxies of some larger-scale potential structures. We show that presence/absence of such a larger-scale potential can consistently account for several unsolved problems with the ISM.

\section{Reference}

Matsushita, K., 1997, PhD thesis, University of Tokyo

Matsushita, K. et al. 1997, ApJL, 488, L125

Matsushita, K. et al. 1997, submitted to Nature 